\title{
Conditional Deletion of Bmal1 Accentuates Microvascular and Macrovascular Injury
}

Ashay D. Bhatwadekar, ${ }^{*}$ Eleni Beli, ${ }^{*}$ Yanpeng Diao, ${ }^{\dagger}$ Jonathan Chen, ${ }^{*}$ Qianyi Luo, ${ }^{*}$ Alpha Alex, ${ }^{*}$ Sergio Caballero, James M. Dominguez, II, ${ }^{*}$ Tatiana E. Salazar, ${ }^{*}$ Julia V. Busik, ${ }^{\S}$ Mark S. Segal, ${ }^{\dagger}$ and Maria B. Grant*

From the Departments of Ophthalmology* and Nephrology, ${ }^{\dagger}$ Indiana University School of Medicine, Indianapolis, Indiana; the Department of Pharmacology and Therapeutics, ${ }^{\ddagger}$ University of Florida, Gainesville, Florida; and the Department of Physiology, ${ }^{\S}$ Michigan State University, East Lansing, Michigan

Accepted for publication

February 16, 2017.

Address correspondence to Maria B. Grant, M.D., Department of Ophthalmology, Eugene and Marilyn Glick Eye Institute, $980 \mathrm{~W}$. Walnut St., Indianapolis, IN 46202; or Ashay D. Bhatwadekar, Ph.D., Department of Ophthalmology, Eugene and Marilyn Glick Eye Institute, 1160 W. Michigan St., GK-318, Indianapolis, IN 46202. E-mail: mabgrant@iupui.edu or abhatwad@iupui.edu.

\begin{abstract}
The brain and muscle aryl hydrocarbon receptor nuclear translocator-like protein (BMAL)-1 constitutes a major transcriptional regulator of the circadian clock. Here, we explored the impact of conditional deletion of Bmal1 in endothelium and hematopoietic cells in murine models of microvascular and macrovascular injury. We used two models of $B m a 1^{\mathrm{fx}} / \mathrm{fx}$; Tek-Cre mice, a retinal ischemia/reperfusion model and a neointimal hyperplasia model of the femoral artery. Eyes were enumerated for acellular capillaries and were stained for oxidative damage markers using nitrotyrosine immunohistochemistry. LSK (lineage-negative, stem cell antigen-1-positive, c-Kit-positive) cells were quantified and proliferation assessed. Hematopoiesis is influenced by innervation to the bone marrow, which we assessed using IHC analysis. The number of acellular capillaries increased threefold, and nitrotyrosine staining increased 1.5-fold, in the retinas of Bmal1 $1^{\mathrm{fx} / \mathrm{fx}} ;$ Tek-Cre mice. The number of LSK cells from the Bmal1 ${ }^{\mathrm{fx} / \mathrm{f}}$ ${ }^{\mathrm{fx}}$;Tek-Cre mice decreased by 1.5 -fold and was accompanied by a profound decrease in proliferative potential. Bmal1 ${ }^{\mathrm{fx} / \mathrm{fx}} ;$ Tek-Cre mice also exhibited evidence of bone marrow denervation, demonstrating a loss of neurofilament-200 staining. Injured femoral arteries showed a $20 \%$ increase in neointimal hyperplasia compared with similarly injured wild-type controls. Our study highlights the importance of the circadian clock in maintaining vascular homeostasis and demonstrates that specific deletion of $B M A L 1$ in endothelial and hematopoietic cells results in phenotypic features similar to those of diabetes. (Am J Pathol 2017, 187: 1426-1435; http://dx.doi.org/10.1016/j.ajpath.2017.02.014)
\end{abstract}

Most organisms possess an endogenous circadian system that drives the daily oscillations of a variety of physiologic and behavioral processes with a 24 -hour period. A variety of cardiovascular and hemodynamic parameters, such as heart rate, blood pressure, endothelial function, and fibrinolytic activity, exhibit such diurnal patterns. ${ }^{1}$ The frequency of occurrence of acute cardiovascular events, such as acute myocardial infarction, myocardial ischemia, cardiac arrest, ventricular tachycardia after myocardial infarction, and sudden death, varies according to the time of day. ${ }^{2,3}$

Although the suprachiasmatic nucleus orchestrates the neuroendocrine physiology of the circadian rhythm, at the molecular level the circadian rhythm is orchestrated by autoregulatory transcriptional/translational loops of a set of clock genes. About $10 \%$ of the transcriptome in all tissues is under the control of these clock genes. ${ }^{4}$ Genetic studies on clock gene-mutant mice recapitulate phenotypic changes observed, similar to those in cardiovascular ${ }^{5}$ and metabolic disorders. ${ }^{6}$ Mice deficient in brain and muscle aryl hydrocarbon receptor nuclear translocator-like protein (Bmal)-1 possess a range of disorders, including reduced lifespan, premature aging, monocytosis, and cataracts.

Diabetes is a multifactorial disease characterized by metabolic abnormalities, including hyperglycemia, insulin

\footnotetext{
Supported by the Ralph and Grace Showalter Trust Fund, International Retinal Research Foundation, an unrestricted award from the Research to Prevent Blindness Foundation to the Department of Ophthalmology, Indiana University (A.D.B.), and NIH grants R01EY007739, R01EY012601, R01NHLBI1 10170, and R01 DK090730 (all to M.B.G). The Flow Cytometry Resource Facility at Indiana University Simon Cancer Center is partially funded by National Cancer Institute grant P30 CA082709.

Disclosures: None declared.
} 
resistance, and an increase in fatty acids, with each abnormality provoking a molecular arsenal that contributes to vascular diseases. ${ }^{7,8}$ Most importantly, there is an interplay between the circadian system and metabolic disorders. ${ }^{9,10}$ A disrupted circadian rhythm in shift workers and frequent flyers leads to an increased susceptibility to metabolic diseases, ${ }^{11}$ and metabolic diseases such as diabetes lead to a disrupted circadian rhythm. ${ }^{12}$

Endothelial dysfunction is central to the underlying pathology of diabetic vascular diseases, along with reduced vascular repair, which is mediated in large part by bone marrow-derived progenitor cells (BMPCs). $\mathrm{We}^{12}$ and others ${ }^{13,14}$ have reported that diabetes is a disease of circadian dysfunction. Our studies using a rat model of type 2 diabetes have demonstrated that core circadian regulatory genes are crucial for the vascular health of the retina ${ }^{12}$ and that dysfunctional release and intrinsic dysfunction of BMPCs contribute to the development of acellular capillaries, the pathologic hallmark of diabetic retinopathy. Using a clock gene (Per2)-mutant mouse model, we demonstrated that a lack of functional period circadian protein homolog-2 recapitulates a vascular phenotype similar to that of diabetic retinopathy. ${ }^{15,16}$ For this study, we asked whether a BMAL1 deficiency in endothelial cells is linked to accelerated microvascular and macrovascular injury. Because the findings from a previous study demonstrated that angiopoietin-1 receptor (TIE-2; alias TEK) is also expressed in a fraction of BMPCs [LSK (lineage-negative, stem cell antigen1 -positive, c-Kit-positive) cells], ${ }^{17}$ we hypothesized that the function of the BMPC population in $\mathrm{Bmall}^{\mathrm{f} / \mathrm{fx}} ; \mathrm{Tek}-\mathrm{Cre}$ mice would also be adversely affected.

\section{Materials and Methods}

\section{Animals}

The animal studies were performed in accordance with the Use of Animals in Ophthalmic and Vision Research statement from the Association for Research in Vision and Ophthalmology. The study was approved by the institutional animal care and use committees at the University of Florida and Michigan State University. The $B m a l^{\mathrm{fx} / \mathrm{fx}_{\mathrm{x}}}$ and Tek-Cre mice were purchased from The Jackson Laboratory (Bar Harbor, ME). The Bmall ${ }^{\mathrm{f} / \mathrm{fx}}$ mice were crossed with Tek-Cre transgenic mice to generate either Bmall ${ }^{\mathrm{fx} / \mathrm{fx}} ;$ TekCre or Bmall ${ }^{\mathrm{f} / \mathrm{fx}}$ controls [wild type (WT)]. All animals were maintained under 12-hour light/dark conditions. All of the studies were performed when the animals were aged between 8 and 10 months.

\section{Ischemia and Reperfusion Injury of the Retina}

The Bmall ${ }^{\mathrm{fx} / \mathrm{fx}} ;$ Tek-Cre and WT mice were kept under inhalational anesthesia. The anterior chamber of the eye was cannulated with a 30-gauge needle attached to an infusion line of sterile saline, and the eye was subjected to 2 hours of hydrostatic pressure in the anterior chamber. After 2 hours, the needle was withdrawn and the intraocular pressure was normalized, resulting in reperfusion injury. Seven days after injury, at which time retinal capillary damage is detectable, the retinas were harvested and processed for trypsin digestion.

\section{Trypsin Digestion of Retinas}

The retinas were processed using a trypsin digestion protocol as described previously. ${ }^{18}$ The retinal digests were mounted on presalinized slides and stained with periodic acid-Schiff base, followed by counterstaining with hematoxylin (Sigma-Aldrich, St. Louis, MO). Acellular capillaries, defined as naked basement membrane tubes lacking both endothelial cells and pericytes, were counted at $\times 20$ magnification by investigators blinded to the respective groups, and the number per square milliliter was quantified.

\section{Immunofluorescence Staining}

The ischemia/reperfusion (I/R)-injured retinas were paraffin embedded and sectioned. After a citrate buffer epitope retrieval, the retinal sections were stained for either endothelial NO synthase (eNOS), Akt, or phospho-Akt antibodies (Cell Signaling, Danvers, MA); Bmall (Novus Biologicals LLC, Littleton, CO); CD31 (Dianova, Hamburg, Germany); and CD45 (Sigma-Aldrich). The immunofluorescence images were quantified for the degree of respective staining using image analysis software (Zen lite version 2; Carl Zeiss Microscopy LLC, Thornwood, NY).

\section{Neointimal Hyperplasia}

The Bmall ${ }^{\mathrm{fx} / \mathrm{fx}} ;$ Tek-Cre and WT mice were kept under inhalational anesthesia. The endoluminal macrovascular injury was performed by introducing a $0.015 \mathrm{~mm}$-diameter angioplasty guidewire into the femoral artery. The animals were euthanized after 4 weeks for the harvesting of the femoral artery. The degree of neointimal hyperplasia was evaluated on transverse sections of the femoral artery by evaluation of the thickness of the luminal area of the injured artery.

\section{Quantification of BMPCs}

Femurs were isolated from $\mathrm{Bmall}^{\mathrm{fx} / \mathrm{fx}} ;$ Tek-Cre and WT mice. The bone marrow was flushed in phosphate-buffered saline containing $2 \%$ fetal bovine serum. After serum blocking for 10 minutes, mononuclear cells were stained with biotin mouse lineage panel (BD Pharmingen, San Diego, CA), followed by staining with Streptavidin APC (eBioscience, San Diego, CA). Stem cell antigen-1 and c-Kit staining were performed using PE-rat anti-mouse Ly-6A/E and PE-Cy7 rat anti-mouse CD117 antibodies (BD Pharmingen). The stained cells were analyzed using an LSR II flow cytometer (BD Biosciences), and data were analyzed using FlowJo software version 9.9.4 (FlowJo, Ashland, OR). 

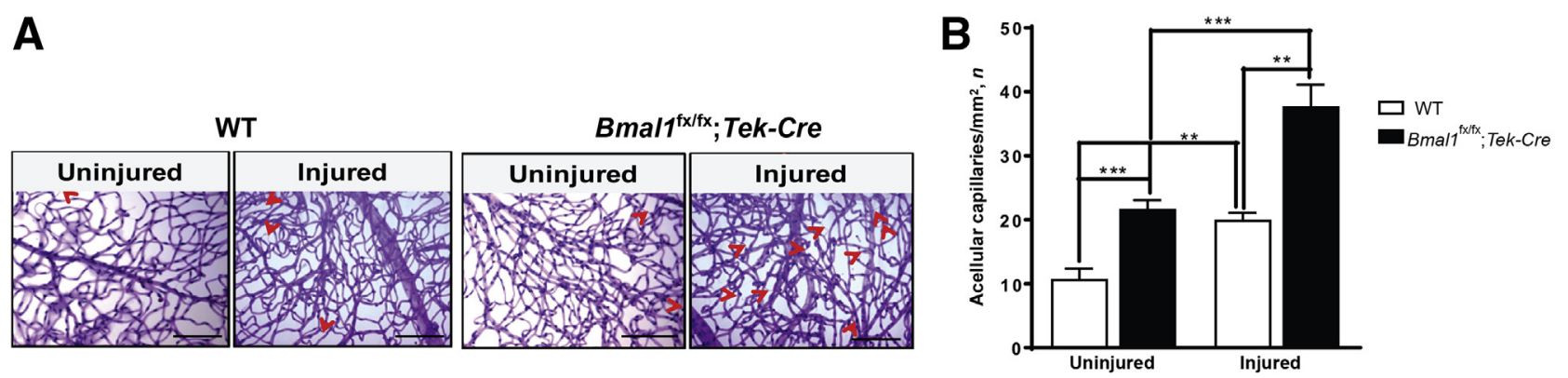

Figure 1 Enhanced development of acellular capillaries in Bmal1 ${ }^{\mathrm{fx} / \mathrm{fx}} ;$ Tek-Cre retinas after ischemia/reperfusion (I/R) injury. Bmal ${ }^{\mathrm{fx} / \mathrm{fx}} ;$ Tek-Cre and WT mice underwent an I/R injury, and 7 days after the injury, the retinas were harvested and trypsin digested. A: Representative images of trypsin digests of untreated or treated eyes from respective groups. Acellular capillaries are indicated by arrowheads. B: Quantification of acellular capillaries. Data are expressed as means \pm SEM. $n=6$ Bmal $^{\mathrm{fx} / \mathrm{fx}} ;$ Tek-Cre; $n=10$ WT. ${ }^{* *} P<0.01,{ }^{* * *} P<0.001$. Scale bars $=100 \mu \mathrm{m}$.

\section{Neurofilament-200 and Tyrosine Hydroxylase Staining of Bone Marrow}

Femurs from Bmall ${ }^{\mathrm{fx} / \mathrm{fx}} ;$ Tek-Cre and WT mice were isolated and embedded in paraffin. Sections were obtained on a microtome at $4 \mu \mathrm{m}$ and stained for tyrosine hydroxylase using polyclonal rabbit anti-tyrosine hydroxylase antibody (EMD Millipore, Billerica, MA), and for neurofilament (NF)-200 using polyclonal rabbit anti-NF-200 antibody (Sigma-Aldrich). The images of stained sections were obtained using an Olympus IX-70 inverted microscope (Olympus, Center Valley, PA).

\section{Protein Estimation in Bone Marrow Supernatants}

The bone marrow was flushed with phosphate-buffered saline; the cellular fraction was pelleted by centrifugation. The supernatant was concentrated using ultracentrifugation filter units (EMD Millipore). The supernatants were analyzed using a Luminex bead-based cytokine array (AssayGate, Ijamsville, MD). The data are shown in picograms of analyte per milligram of total protein.

\section{Statistical Analysis}

The data are expressed as means \pm SEM. Statistical analysis was performed using Prism software version 6 (GraphPad Software, La Jolla, CA), and data were analyzed using oneway analysis of variance followed by the Tukey-Kramer post hoc test or $t$-test.

\section{Results}

Decrease in Bmal1 Staining in Endothelial Cells of Bmal1 ${ }^{\mathrm{fx} / \mathrm{fx}}$;Tek-Cre Mice

To confirm endothelial deletion of Bmall, we stained retinal sections with Bmal1 and CD31 antibodies
A

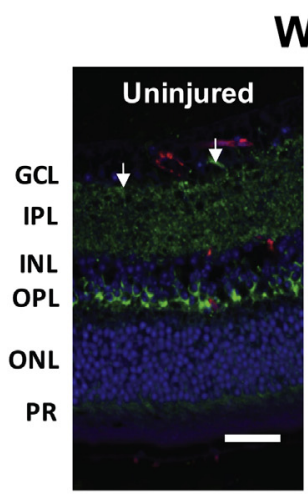

WT

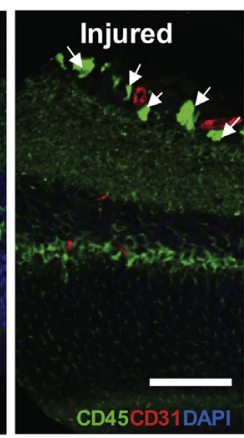

\section{Bmal1fx/fx; Tek-Cre}

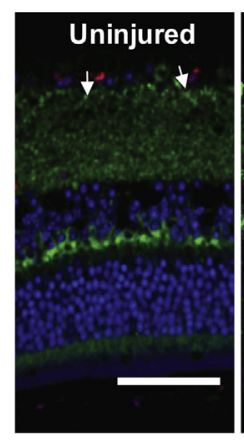

B

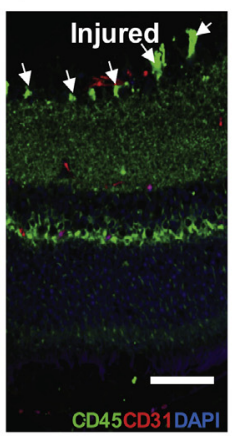

B $\square$ wт

$B m a l 1^{\mathrm{fx} / \mathrm{fx}} ;$ Tek-Cre

Figure 2 Increased leukocytes in ischemia/reperfusion (I/R)-injured retinas. The retinas of I/R-injured Bmal ${ }^{\mathrm{fx} / \mathrm{fx}}$; Tek-Cre and WT mice were stained with CD45-Alexa 488 and CD31-Alexa 555 antibodies. A: Representative images of retinal sections showing an increase in CD45 stain (arrows) in injured retinas. B: Quantification of CD45 clusters. Data are expressed as means \pm SD. $n=3$ per group. ${ }^{* * P} P 0.01,{ }^{* *} P<0.001$. Scale bars $=50 \mu \mathrm{m}$. GCL, ganglion cell layer; INL, inner nuclear layer; IPL, inner plexiform layer; ONL, outer nuclear layer; OPL, outer plexiform layer; PR, photoreceptor. 
A

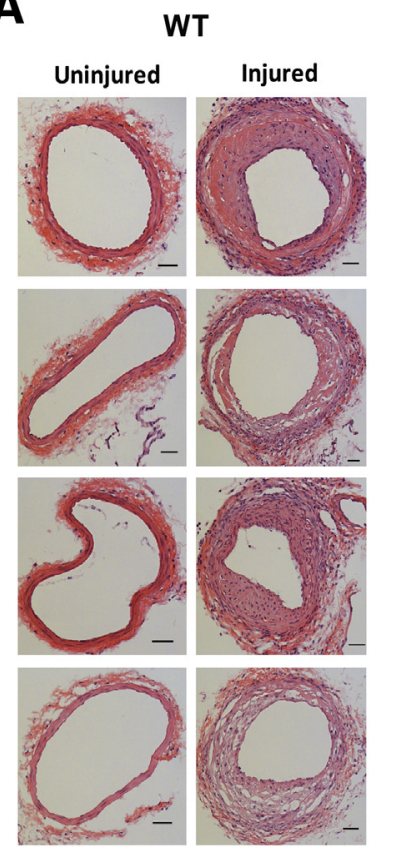

B

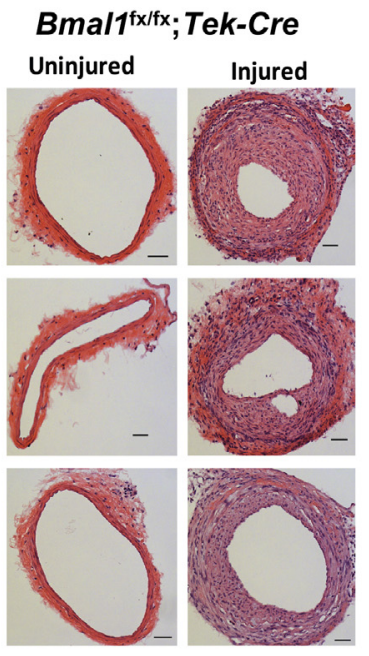

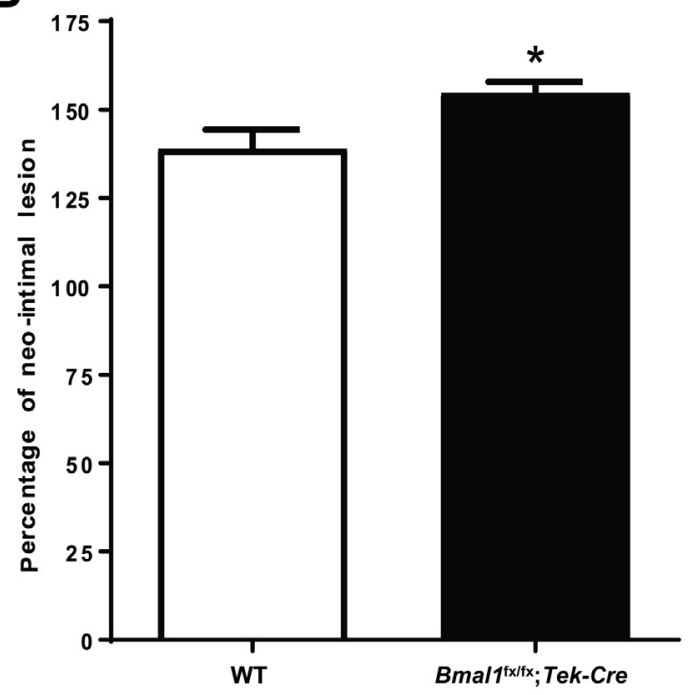

Figure 3 Increase in neointimal hyperplasia in $B m a l 1^{\mathrm{fx} / \mathrm{fx}} ;$ Tek-Cre mice. Endoluminal macrovascular injury was performed into the femoral artery, and the degree of neointimal hyperplasia was evaluated 4 weeks after injury. A: Transverse sections of WT and Bmal- ${ }^{\mathrm{fx} / \mathrm{fx}}$; Tek-Cre femoral arteries, showing a degree of

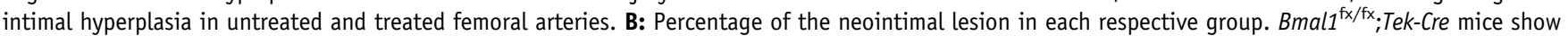

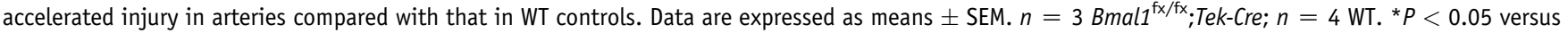
WT. Scale bars $=20 \mu \mathrm{m}$.

(platelet endothelial adhesion molecule-1, an endothelial marker). Bmall staining was observed in the ganglion cell layer, inner nuclear layer, and outer plexiform layer. However, the vessels of Bmall ${ }^{\mathrm{fx} / \mathrm{fx}} ; T e k$-Cre mice exhibited a marked decrease in staining for Bmall compared with that in WT controls (Supplemental Figure S1).

\section{$\mathrm{Bmal1}^{\mathrm{fx} / \mathrm{fx}} ;$ Tek-Cre Mice Demonstrate an Increase in Acellular Capillaries}

Increased numbers of acellular capillaries represent the hallmark feature of ischemic and diabetic retinopathies. In the acute model of $I / R$ injury used in this study, capillary degeneration mimics that observed in diabetic retinopathy. ${ }^{19}$ Specifically, the I/R model recapitulates the small vessel disease seen in diabetes, but without the long time course required for diabetic eye changes and without the confounding metabolic effects associated with diabetes. ${ }^{19}$ WT eyes that underwent retinal I/R injury exhibited an increase (1.86-fold; $P<0.01)$ in acellular capillaries compared with those in control uninjured eyes. The uninjured Bmall ${ }^{\mathrm{fx} / \mathrm{fx}}$;Tek-Cre eyes demonstrated retinal pathology under baseline conditions, as they had 2.07-fold more $(P<0.001)$ acellular capillaries compared with those in WT controls. However, after I/R injury, the $B m a l l^{\mathrm{fx} / \mathrm{fx}}$; Tek-Cre mice showed a dramatic increase in retinal damage compared with that in the injured WT mice. The rate of the fold increase in acellular capillaries was accelerated, showing 2.28-fold in the Bmall ${ }^{\mathrm{fx} / \mathrm{fx}} ;$ Tek-Cre mice compared with 1.31-fold in WT mice $P<0.001$ (Figure 1).

\section{Increase in $\mathrm{CD}_{4} 5^{+}$Leukocytes in Bmal- ${ }^{\mathrm{fx} / \mathrm{fx}} ;$ Tek-Cre Mice}

To explore any leukocyte-mediated involvement in the I/R injury, we stained retinal cross sections with $\mathrm{CD} 45^{+}$antibodies (a pan-leukocyte marker) and CD31 antibodies (platelet endothelial adhesion molecule-1, an endothelial marker). With $\mathrm{I} / \mathrm{R}$ injury in WT controls, there was a 2.9-fold $(P<0.001)$ increase in leukocytes compared with those in uninjured WT-controls. In Bmall ${ }^{\mathrm{fx} / \mathrm{fx}} ;$ TekCre mice, the increase was also 2.9 -fold $(P<0.01)$ compared with that in the uninjured Bmall ${ }^{\mathrm{fx} / \mathrm{fx}} ;$ Tek-Cre mice. There was no significant difference in leukocyte numbers between WT and Bmall ${ }^{\mathrm{fx} / \mathrm{fx}} ;$ Tek-Cre mice (Figure 2).

\section{Bmal1 $^{\mathrm{fx} / \mathrm{fx}} ;$ Tek-Cre Mice Exhibit an Increase in Neointimal Hyperplasia}

To explore whether Bmal1 deficiency adversely affects the macrovasculature, we used an animal model of neointimal hyperplasia. Four weeks after the injury, the femoral artery was assessed for degree of neointimal hyperplasia. The aortas of Bmall ${ }^{\mathrm{f} / \mathrm{fx}} ;$ Tek-Cre mice exhibited thicker walls compared with those in injured WT mice $(P<0.05)$ (Figure 3). 

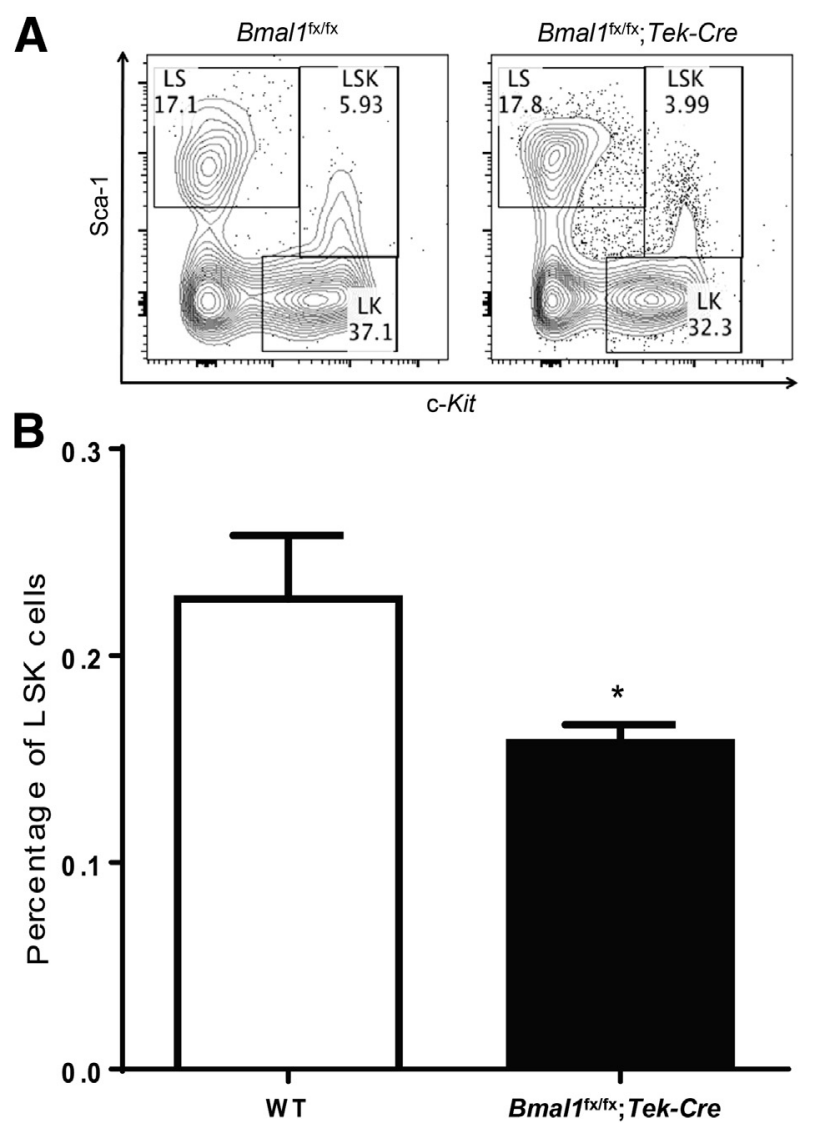

Figure 4 Decrease in LSK cells in $\mathrm{Bmal1}^{\mathrm{fx} / \mathrm{fx}} ;$ Tek-Cre mice. The bone marrow from WT and Bmal1 ${ }^{\mathrm{fx} / \mathrm{fx}}$; Tek-Cre mice were harvested, and the percentages of LSK cells were determined using flow cytometry. A: A representative flow cytometry dot plot showing selecting gating strategy used for separate the LSK population. B: Decreased LSK cells in Bmal1 ${ }^{\mathrm{fx} / \mathrm{fx}}$; Tek-Cre mice. Data are expressed as means \pm SEM. $n=5$ per group. ${ }^{*} P<0.05$ versus WT. LK, lineage and c-Kit; LS, lineage and sca-1; LSK, lineage-negative, stem cell antigen-1-positive, c-Kit-positive.

\section{Decrease in Bmal1 Staining in Bone Marrow}

We next characterized the expression of Bmall protein in bone marrow by staining the femurs of Bmall ${ }^{\mathrm{fx} / \mathrm{fx}} ;$ Tek-Cre mice with Bmall antibodies. We observed a marked decrease in Bmall staining in bone marrow sections of Bmall $^{\mathrm{fx} / \mathrm{fx}} ;$ Tek-Cre mice (Supplemental Figure S2).

\section{Bmal1 $^{\mathrm{fx} / \mathrm{fx}} ;$ Tek-Cre Mice Demonstrate Decreased Levels} of LSK Cells

BMPCs play an important role in vascular repair by expression of growth factors and cytokines needed by the injured endothelium. TEK is expressed in a fraction of LSK cells, which have the ability to generate long-term, reconstituting, colony-forming cells. ${ }^{17}$ Thus, we reasoned that functional deletion of BMAL1 in LSK cell fraction will adversely affect the LSK cell numbers, leading to compromised vascular repair. LSK numbers were quantitated using flow cytometry, and after omission of the lineage, negative fraction, stem cell antigen- $1^{+}$, and $\mathrm{c}-\mathrm{Kit}^{+}$, they were enumerated as dual-positive cells (Figure 4A). The Bmall $^{\mathrm{fx} / \mathrm{fx}} ;$ Tek-Cre mice demonstrated a significant decrease (1.46-fold; $P<0.05)$ in the percentage of LSK cell numbers compared with that in WT control mice (Figure 4B).

\section{Decrease in Stem Cell Mobilization Factors in the Bone} Marrow of $\mathrm{Bmal1}^{\mathrm{fx} / \mathrm{fx}}$;Tek-Cre Mice

To identify a possible mechanism for the decrease in LSK cell numbers, we analyzed the bone marrow supernatants using a multiplex array panel. We observed that, with a lack of Bmall in bone marrow (Bmall ${ }^{\mathrm{f} / \mathrm{fx}} ;$ Tek-Cre mice), there was a 1.8-fold $(P<0.05)$ decrease in granulocyte-monocyte colony stimulating factor, a slight decrease in stem cell factor, and a marginal elevation in stromal derived factor-1 or chemokine (C-X-C motif) ligand-12 (Supplemental Figure S3).

Notably, we observed decreases in a variety of inflammatory markers, such as tumor necrosis factor- $\alpha$, IL-6, IL- $1 \alpha$, IL- $1 \beta$, and a macrophage activator, interferon- $\gamma$. There was a nonsignificant increase in macrophage inflammatory protein-2 (1.2-fold) or chemokine (C-X-C motif) ligand-2 (Supplemental Figure S4).

\section{Bmal-1 ${ }^{\mathrm{fx} / \mathrm{fx}} ;$ Tek-Cre Mice Show Decreased Proliferation of BMPCs}

To further characterize the bone marrow cell defect, the proliferative capacity of the mononuclear fraction was evaluated using methylcellulose colony forming units (CFUs). The potential for proliferation of BMPCs from the Bmall ${ }^{\mathrm{fx} /}$ ${ }^{\mathrm{fx}}$;Tek-Cre mice was markedly reduced, as evidenced by reductions in CFUs, specifically a threefold decrease $(P<0.05)$ in CFU-granulocyte-monocytes (Figure 5A); a fourfold $(P<0.05)$ decrease in $\mathrm{CFU}$-granulocyte-erythrocytemonocyte/macrophage-megakaryocytes (Figure 5C); and a sixfold decrease $(P<0.05)$ in $\mathrm{CFU}$-granulocytes (Figure 5D), but a nonsignificant increase in CFUmonocytes (Figure 5B).

\section{Retinas of Bmal1 ${ }^{\mathrm{fx} / \mathrm{fx}}$; Tek-Cre Mice Show an Increase in Nitrotyrosine Staining}

To identify potential mechanism(s) for the increased microvascular injury observed in the Bmall ${ }^{\mathrm{fx} / \mathrm{fx}} ;$ Tek-Cre mice, the levels of oxidative injury in the injured retinas were examined. In the presence of the reactive oxidant species, such as superoxides, nitric oxide (NO) rapidly forms peroxynitrite. The major product of the reaction of proteins with peroxynitrite is a nitro group added to tyrosine to form nitrotyrosine. We reasoned that the combination of a lack of Bmal1 and I/R injury would result in an increase in the levels of nitrotyrosine. The retinas of uninjured WT mice exhibited minimal nitrotyrosine staining, whereas nitrotyrosine was markedly increased in $\mathrm{I} / \mathrm{R}$-injured WT retinas (Figure 6A). The increase in fluorescence intensity 

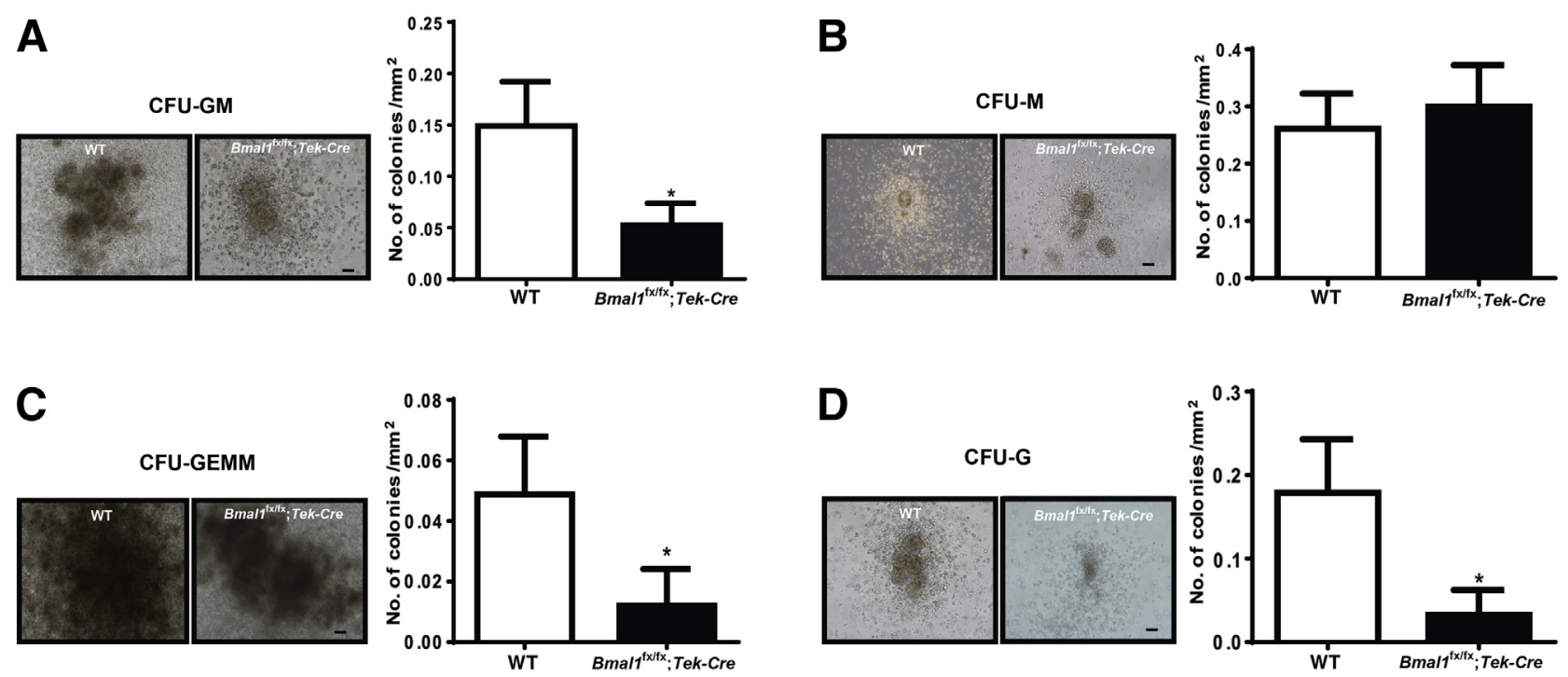
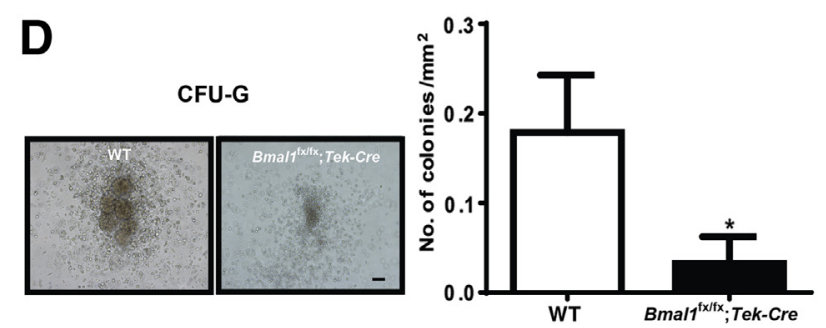

Figure 5 Decreased proliferation in Bmal1 ${ }^{\mathrm{fx} / \mathrm{fx}} ;$;ek-Cre mice. Bone marrow mononuclear cells were isolated from WT and Bmal-1 ${ }^{\mathrm{fx} / \mathrm{fx}} ;$ Tek-Cre mice and were propagated in methylcellulose semisolid cultures; the number of colonies was enumerated after one week. Representative images and bar charts showing decreases in CFU-GM (granulocyte monocyte) (A), CFU-GEMM (granulocyte, erythrocyte, monocyte/macrophage, megakaryocyte) (C), and CFU-G (granulocyte) (D), and an increase in CFU-monocytes (M) in Bmal- ${ }^{\mathrm{fx} / \mathrm{fx}}(\mathbf{B})$. Data are expressed as means \pm SEM. $n=5$ WT; $n=6$ Bmal ${ }^{\mathrm{f} \times / \mathrm{fx}} ;$ Tek-Cre. $^{*} P<0.05$ versus WT. Scale bars $=200 \mu \mathrm{m}$.

was observed in the outer plexiform layer, consisting of synapses of horizontal cells, bipolar cells, and photoreceptor inner segments, and in the inner plexiform layer, consisting of synapses of amacrine cells and ganglion cells. Uninjured retinas from Bmall $^{\mathrm{fx} / \mathrm{fx}} ;$ Tek-Cre mice demonstrated increased nitrotyrosine compared with that in uninjured WT retinas, and nitrotyrosine staining was markedly intensified in the I/R-injured Bmal1 ${ }^{\mathrm{fx} / f \mathrm{x}}$; Tek-Cre mice (Figure 6A). Quantification of fluorescence intensity demonstrated a 1.5fold increase $(P<0.05)$ in injured Bmall ${ }^{\mathrm{f} / \mathrm{fx}} ; \mathrm{Tek}$-Cre retinas compared with that in WT injured retinas (Figure 6B).

\section{Bmal1 $^{\mathrm{fx} / \mathrm{fx}} ;$ Tek-Cre Mice Exhibit Bone Marrow Pathology}

We previously reported that bone marrow neuropathy adversely affected BMPC function and release into the circulation in a model of type 2 diabetes. ${ }^{12}$ The bone marrow neuropathy was mediated by neuronal damage due to a dysfunctional vasa nervorum. We reasoned that a lack of BMAL1 in the vascular endothelium of the vasa nervorum would induce a neuropathic phenotype resulting in compromised BMPC functioning. Femurs were stained and quantified for NF-200 and tyrosine hydroxylase. The NF200 staining of the bone marrow was decreased by 1.5 -fold $(P<0.01)$ (Figure $7 \mathrm{~A})$; however, the tyrosine hydroxylase staining remained unchanged (Figure $7 \mathrm{~B}$ ).

\section{$\mathrm{Bmal}^{\mathrm{fx} / \mathrm{fx}} ;$ Tek-Cre Mice Exhibit Defects in Akt and eNOS Signaling in the Retina}

Activation of the serine-threonine kinase Akt represents a crucial prosurvival signal for endothelial cells by increasing NO generation via phosphorylation of eNOS. We asked whether the lack of BMAL1 could lead to a reduction in expression of AKT, phospho-AKT, or eNOS immunoreactivity. Retinal sections were stained for eNOS, Akt, and phospho-Akt, and the intensity of staining around blood vessels was evaluated using image morphometry. Profound decreases in the expressions of total Akt (Figure 8A) and phospho-Akt (Figure 8B) $(P<0.05)$, but not of eNOS (Figure $8 \mathrm{C}$ ), were observed in the retinal vessels from $\mathrm{Bmal}^{\mathrm{fx} / \mathrm{fx}} ;$ Tek-Cre mice.

\section{Discussion}

It is widely appreciated that the biological clock interacts with environmental cues to influence a variety of cardiovascular and hemodynamic parameters. The findings from our study demonstrate that a loss of endothelial BMAL1 orchestrates the progression of microvascular and macrovascular damage. In agreement with findings from a previous study by Anea et al, ${ }^{20}$ which reported endothelial dysfunction in whole-body Bmall-knockout mice, the findings from our study support the concept that endothelial Bmall is necessary for vascular health. In our study, the conditional deletion of Bmall in vascular endothelium accelerated both retinal microvascular and femoral arterial macrovascular injury. The lack of Bmal1 in BMPCs further aggravated endothelial injury due to the inability of these cells to adequately participate in vascular repair.

In this study, we generated a conditional knockout mouse using the Tie2-Cre (Tek-Cre) transgene. In this animal model, the mouse endothelial-specific receptor, tyrosine kinase (Tie-2 or Tek) promoter directed the expression of Cre recombinase. The TIE-2 gene (TEK) encodes an 

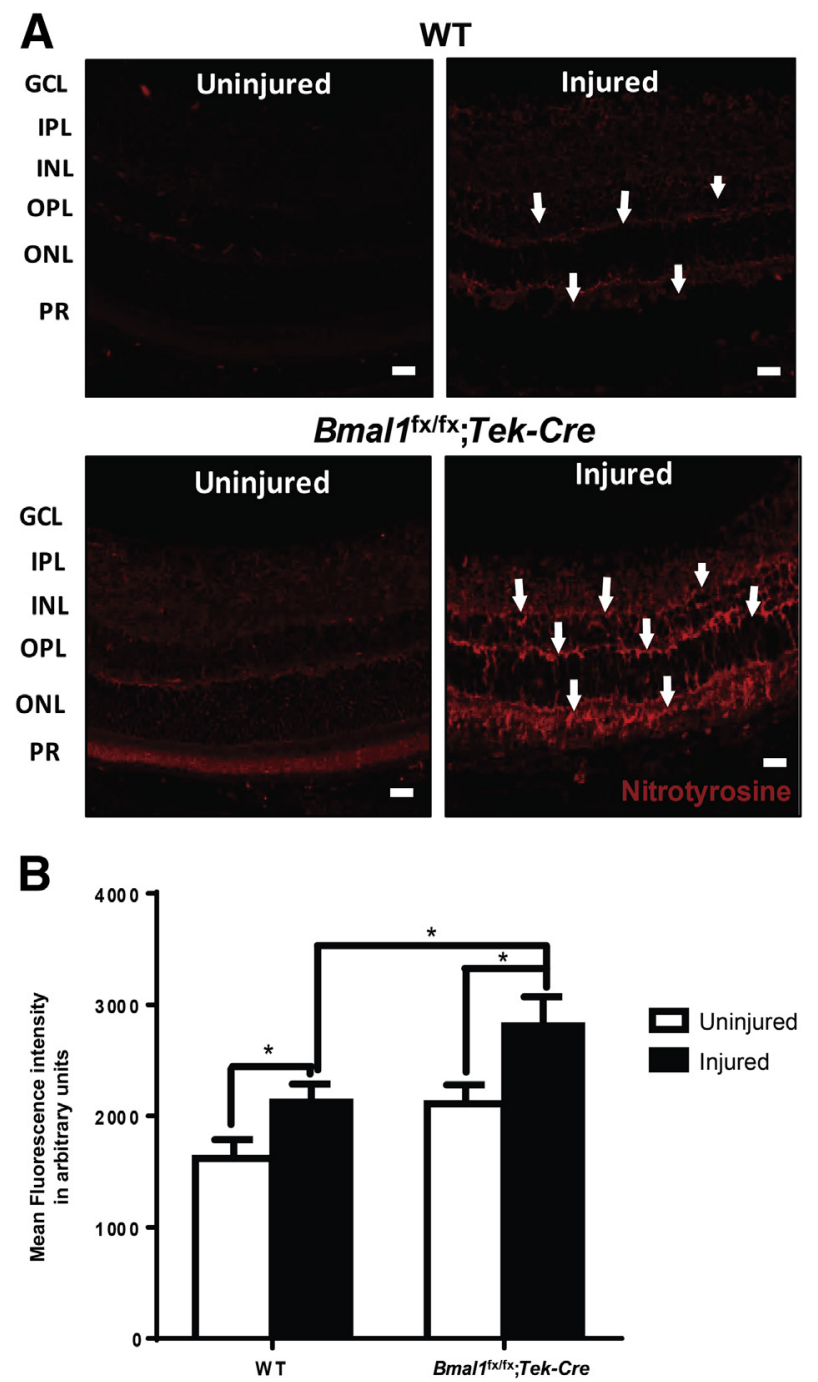

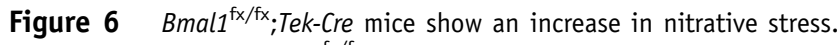
The retinas of WT and $\mathrm{Bmal}^{\mathrm{f} \times \mathrm{fx}}$; $T$ Tek-Cre mice after an ischemia/reperfusion injury were paraffin-embedded, sectioned, and stained for nitrotyrosine. A: Representative images of retinal mounts showing a profound increase in nitrotyrosine staining in injured Bmall ${ }^{\mathrm{f} / \mathrm{fx}} ;$ Tek-Cre mice (arrows). B: Quantification fluorescence intensity for nitrotyrosine. Data are expressed as means \pm SEM. $n=5 \mathrm{WT} ; n=6 \mathrm{Bmal}^{\mathrm{f} \times / \mathrm{fx}}$; Tek-Cre. ${ }^{*} P<0.05$. Scale bars $=20 \mu \mathrm{m} . \mathrm{GCL}$, ganglion cell layer; INL, inner nuclear layer; IPL, inner plexiform layer; $\mathrm{ONL}$, outer nuclear layer; $\mathrm{OPL}$, outer plexiform layer; PR, photoreceptor layer.

angiopoietin receptor, a member of the receptor tyrosine kinase family. ${ }^{21}$ Murine Tie-2 expression is detected as the first endothelial cells arise, remains positive in endothelial cells throughout development, and is detectable in virtually all endothelial cells in adult tissues. ${ }^{22-24}$ However, there are reports that TIE-2 is also expressed in subpopulations of BMPCs. ${ }^{25}$ Thus, we considered that the crossing of these animals with $\mathrm{Bmal}^{\mathrm{f} / \mathrm{fx}}$ mice generated a conditional knockout mouse specifically lacking Bmal1 in endothelial cells and BMPCs. The loss of BMAL1 in LSK cells and BMPCs, as well as in endothelial cells, affects their functioning, and endothelial dysfunction would lead to worsening neointimal hyperplasia. The neointimal process is affected by endothelial repair, and monocytes infiltrate (early on), but by 4 weeks the majority of the cells within the lesion are smooth muscle cells. Although the degree of intimal hyperplasia was only a $20 \%$ increase, this was in addition to a robust $135 \%$ neointimal lesion. The findings from our study suggest that retinal deterioration was increased at baseline due to the endothelial loss of the Bmall. As the studies were performed in animals 8 to 10 months of age, we cannot rule out that these changes were not partially developmental. Additional studies using younger animals could elucidate this further.

The eyes of the Bmall $^{\mathrm{fx} / \mathrm{fx}} ;$ Tek-Cre mice that underwent I/ $\mathrm{R}$ injury exhibited an accelerated increase in retinal acellular capillaries 7 days after injury compared with that in the WT controls. Findings from a previous study suggest that an increase in acellular capillaries in I/R-injured retinas is due to enhanced expression of inducible NOS. ${ }^{26}$ In our study, the I/R-injured retinas from $\mathrm{Bmall}^{\mathrm{fx} / \mathrm{fx}} ;$ Tek-Cre mice were stained intensely for nitrotyrosine, suggesting that NO signaling plays a crucial role in mediating the observed injury response in these mice. NO generated by glutamate accumulates in diabetes ${ }^{27}$ and plays an important role in the degeneration of retinal capillaries. In the presence of superoxide, NO rapidly forms a strong oxidant peroxynitrite that rapidly interacts with proteins, resulting in the formation of nitrotyrosine. ${ }^{27} \mathrm{We}$ speculate that $\mathrm{NO}$ and BMAL1 form a tight regulatory loop, wherein both components are closely dependent on each other. Anea et $\mathrm{al}^{28}$ previously reported that the whole-body Bmall knockout mice exhibit aberrant vascular remodeling and enhanced susceptibility to thrombosis in the carotid arteries of older animals. They showed that a lack of BMAL1 results in increased generation of superoxide radical. ${ }^{28}$ We believe our data support their work, as we observed an increase in retinal nitrotyrosine expression in Bmall $^{\mathrm{fx} / \mathrm{fx}} ;$ Tek-Cre mice both at baseline and after I/R injury. An increase in nitrotyrosine staining in retinas from $\mathrm{Bmall}^{\mathrm{f} / \mathrm{fx}} ; \mathrm{Tek}$-Cre mice was not only limited to retinal vasculature but also prominent in retinal neuropile.

The vessel remodeling in the whole-body Bmall knockout mice was linked to a decrease in Akt and eNOS activity. Although not tested in our macrovascular injury model, we believe that the increase in neointimal hyperplasia observed in the Bmall ${ }^{\mathrm{fx} / \mathrm{fx}} ; \mathrm{Tek}$-Cre mice may be similarly related to a decrease in eNOS and Akt activity. ${ }^{29}$ Immunofluorescence studies on retinal sections further support the involvement of eNOS-AKT signaling by the exaggerated injury response observed in $\mathrm{Bmall}^{\mathrm{fx} / \mathrm{fx}} ; \mathrm{Tek}$-Cre mice. Findings from studies suggest that AKT is a putative target of the circadian clock, ${ }^{30}$ and that the expression of Akt1 is substantially reduced in Bmall knockout mice. ${ }^{20}$ It remains unclear how BMAL1 promotes the AKT activity; one plausible explanation is that the AKT signaling may be directly under the clock regulation. Another possibility is that BMAL1 directly affects the protein abundance of RICTOR (rapamycin-insensitive companion of mammalian 


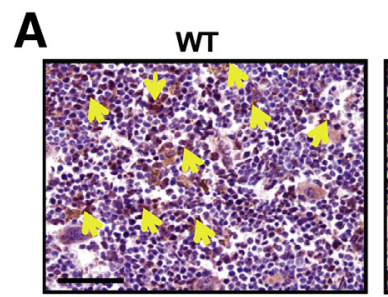

B

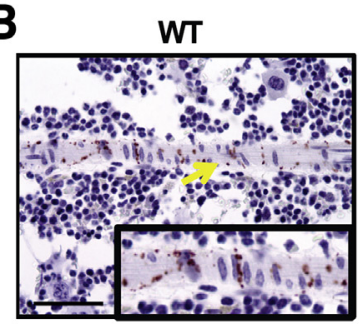

$B m a l 1^{\mathrm{fx} / \mathrm{fx}} ;$ Tek-Cre
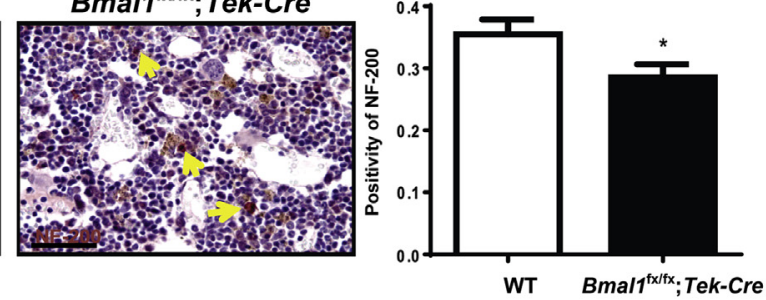

$B m a l 1^{\mathrm{fx} / \mathrm{fx}} ;$ Tek-Cre

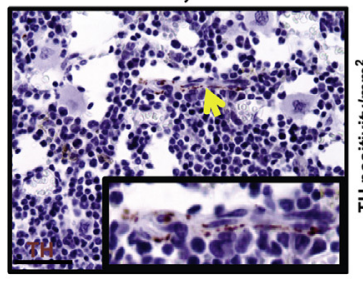

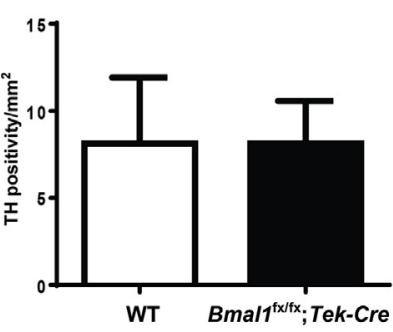

Figure 7 Bone marrow neuropathy in $\mathrm{Bmal1}^{\mathrm{fx} / \mathrm{fx}}$; Tek-Cre mice. Decalcified bones were sectioned and stained for tyrosine hydroxylase (TH) and neurofilament (NF)-200. Representative images showing a decrease in NF-200 (A, arrows) and TH (B, arrows). Insets show $\mathrm{TH}$-positive areas at higher magnification. Bar chart showing quantification of TH and NF200 staining. Data are expressed as means \pm SEM. $n=5$ per group. ${ }^{*} P<0.05$ versus WT. Scale bars $=50 \mu \mathrm{m}$. target of rapamycin), a key component of mTORC2 (mammalian target of rapamycin complex 2), known to phosphorylate AKT2 at phospho-AKT (SER-473) in the liver. However, further studies are warranted to explain the involvement of BMAL1 in AKT signaling within the retina.

The findings from our study also suggest that the BMPC dysfunction due to the conditional deletion of BMALI is detrimental to the retinal and femoral artery vasculature and highlights the crucial role of BMAL1 in maintaining BMPC health. The Bmall ${ }^{\mathrm{fx} / \mathrm{fx}} ;$ Tek-Cre mice showed a profound decrease in BMPC numbers and proliferation. Findings from a previous study suggest that, in the whole-body Bmall knockout animals, there exists a loss of diurnal rhythm in circulating progenitor cells and a decrease in levels of the cytokine chemokine (C-X-C motif) ligand-12, necessary for BMPC release from the bone marrow. ${ }^{31}$ However, we did not observe a significant change in chemokine (C-X-C motif) ligand-12 in our conditional knockout animals. Instead, we
A

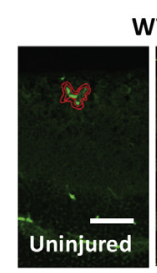

WT

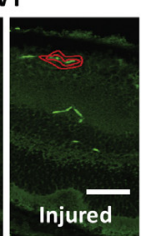

$\mathbf{B}$

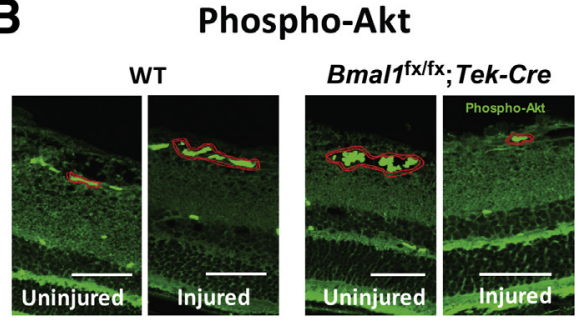

C

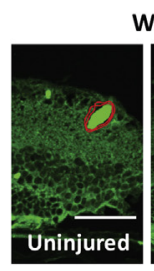

eNOS

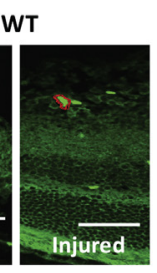

Akt

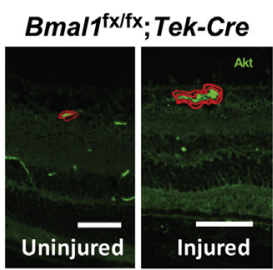

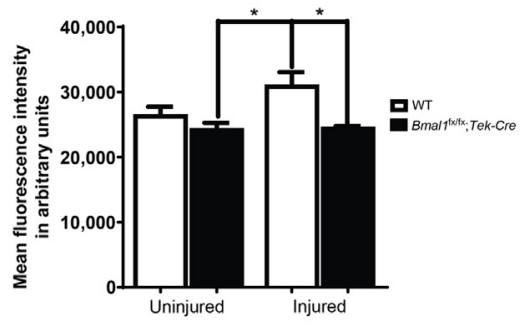
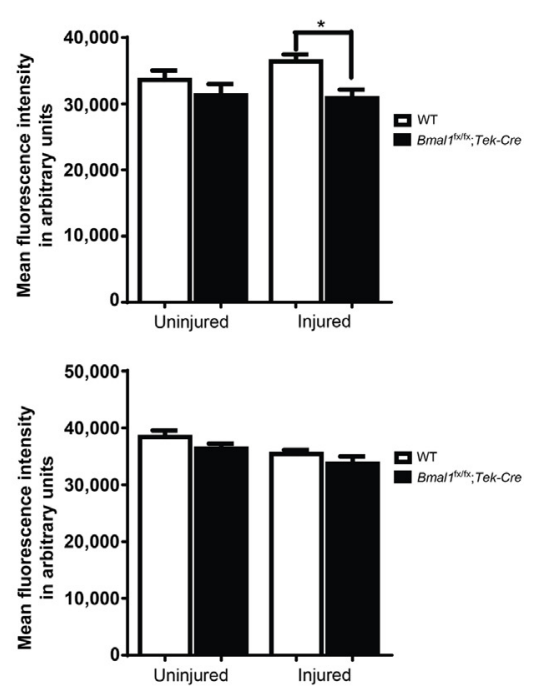

Figure 8 Decrease in Akt-endothelial NO synthase (eNOS) signaling in retinal vasculature of $B m a l 1^{\mathrm{fx} / \mathrm{fx}}$;Tek-Cre mice. The retinal sections of WT and Bmal1 ${ }^{\mathrm{fx} / \mathrm{fx}_{\mathrm{x}}}$;Tek-Cre stained with Akt (A), phospho-Akt (B), and eNOS (C) antibodies. Representative photomicrographs show a staining pattern of respective antibodies. The degree of staining in the area around the blood vessel representing vascular endothelium (highlighted in red with brightly fluorescent red blood cells) was quantified using image analysis software (Zen 2 lite). Bar charts show quantification of intensity for Akt, phospho-Akt, and endothelial NO synthase (eNOS). Data are expressed as means \pm SEM. $n=3$ per group. ${ }^{*} P<0.05$. Scale bars $=50 \mu \mathrm{m}$. 
observed a profound decrease in granulocyte-monocyte colony stimulating factor, which is secreted by a variety of cell types, such as macrophages, T cells, mast cells, NK cells, endothelial cells, and fibroblasts and is known for its pleiotropic effects. We speculate that the decrease in granulocyte-monocyte colony stimulating factor may be attributed to the decrease in LSK cell numbers. In the Bmall ${ }^{\mathrm{fx} / \mathrm{fx}} ;$ Tek-Cre mice, we observed significant decreases in interferon- $\gamma$, tumor necrosis factor- $\alpha$, IL-6, IL- $1 \alpha$, and IL-1 $\beta$ compared with those in controls. We propose that these changes are due to a loss of mesenchymal stem cells, cells involved in the generation of these cytokines. Furthermore, there is a loss of innervation in the mesenchymal stem cells, reducing the generation and release of cytokines and growth factors from these cells. ${ }^{32-34}$ The loss of innervation is supported by the reduced levels of NF-200 immunostaining (Figure 7A). Thus, the reduction of these inflammatory factors supports longstanding bone marrow dysfunction in these mice and warrants further investigation.

Our study also showed decreases in CFU-granulocytemonocytes, CFU-granulocyte-erythrocyte-monocyte/macrophage-megakaryocytes, and CFU-granulocytes, and an increase in CFU-monocytes, due to the conditional deletion of Bmall. We believe that the decrease in BMPC numbers is, in part, due to bone marrow denervation, as indicated by an overall decrease in NF-200 staining. Bone marrow neuropathy results in specific denervation of bone marrow stromal cells, which in turn results in a change in the expression of the key cytokines and growth factors needed for hematopoietic stem cell-progenitor cell differentiation and function. However, in our studies, there was a down-regulation of several cytokines that are known to be increased with inflammation. One possible explanation for an increase in CFU-monocytes is that TIE-2 expression in BMPCs is required for maintaining quiescence and maintaining adherence in the osteoblastic niche of the bone marrow. ${ }^{25}$ The findings from our study support the concept that a lack of BMAL1 in TIE-2-expressing BMPCs alters the quiescent stage of hematopoietic stem cells, leading to an increase in myelomonocytic (CFUmonocyte) cells in the bone marrow. Additional studies are necessary for further characterizing bone marrow defects in $\mathrm{Bmall}^{\mathrm{fx} / \mathrm{fx}} ;$ Tek-Cre mice.

In conclusion, the findings from our study highlight the importance of the circadian clock system in maintaining vascular homeostasis, and that endothelial- and BMPCspecific deletion of BMAL1 is detrimental to both microand macrovasculature beds and results in denervation of the bone marrow, leading to BMPC dysfunction.

\section{Acknowledgment}

We thank the Flow Cytometry Resource Facility at Indiana University Simon Cancer Center (Indianapolis, IN).

E.B. helped with the flow cytometry analysis; D.Y. performed experiments involving the femoral artery-injury model; J.C., Q.L., and A.A. performed immunofluorescence staining of the retinal sections and analyzed the data; S.C., J.M.D., and T.E.S. helped with the animal studies; J.V.B. provided the $\mathrm{Bmal}^{\mathrm{fx} / \mathrm{fx}} ;$ Tek-Cre mice; M.S.S. supervised the studies involving the femoral artery-injury model; A.D.B. and M.B.G. were involved in the conception and design of the study, collection, analysis, and interpretation of the data, and the writing, financial support, and final approval of the manuscript.

\section{Supplemental Data}

Supplemental material for this article can be found at http://dx.doi.org/10.1016/j.ajpath.2017.02.014.

\section{References}

1. Reilly DF, Westgate EJ, FitzGerald GA: Peripheral circadian clocks in the vasculature. Arterioscler Thromb Vasc Biol 2007, 27:1694-1705

2. Muller JE, Stone PH, Turi ZG, Rutherford JD, Czeisler CA, Parker C, Poole WK, Passamani E, Roberts R, Robertson T, Sobel BE Willerson JT, Braunwald E; MILIS Study Group: Circadian variation in the frequency of onset of acute myocardial infarction. N Engl J Med 1985, 313:1315-1322

3. Willich SN: Circadian variation and triggering of cardiovascular events. Vasc Med 1999, 4:41-49

4. Miller BH, McDearmon EL, Panda S, Hayes KR, Zhang J, Andrews JL, Antoch MP, Walker JR, Esser KA, Hogenesch JB, Takahashi JS: Circadian and CLOCK-controlled regulation of the mouse transcriptome and cell proliferation. Proc Natl Acad Sci U S A 2007, 104:3342-3347

5. Young ME, Razeghi P, Taegtmeyer H: Clock genes in the heart: characterization and attenuation with hypertrophy. Circ Res 2001, 88: $1142-1150$

6. Turek FW, Joshu C, Kohsaka A, Lin E, Ivanova G, McDearmon E, Laposky A, Losee-Olson S, Easton A, Jensen DR, Eckel RH, Takahashi JS, Bass J: Obesity and metabolic syndrome in circadian Clock mutant mice. Science 2005, 308:1043-1045

7. Creager MA, Luscher TF, Cosentino F, Beckman JA: Diabetes and vascular disease: pathophysiology, clinical consequences, and medical therapy: part I. Circulation 2003, 108:1527-1532

8. Luscher TF, Creager MA, Beckman JA, Cosentino F: Diabetes and vascular disease: pathophysiology, clinical consequences, and medical therapy: part II. Circulation 2003, 108:1655-1661

9. Staels B: When the Clock stops ticking, metabolic syndrome explodes. Nat Med 2006, 12:54-55; discussion 5

10. Laposky AD, Bradley MA, Williams DL, Bass J, Turek FW: Sleepwake regulation is altered in leptin-resistant $(\mathrm{db} / \mathrm{db})$ genetically obese and diabetic mice. Am J Physiol Regul Integr Comp Physiol 2008, 295:R2059-R2066

11. Zee PC, Attarian H, Videnovic A: Circadian rhythm abnormalities. Continuum (Minneap Minn) 2013, 19:132-147

12. Busik JV, Tikhonenko M, Bhatwadekar A, Opreanu M, Yakubova N, Caballero S, Player D, Nakagawa T, Afzal A, Kielczewski J, Sochacki A, Hasty S, Li Calzi S, Kim S, Duclas SK, Segal MS, Guberski DL, Esselman WJ, Boulton ME, Grant MB: Diabetic retinopathy is associated with bone marrow neuropathy and a depressed peripheral clock. J Exp Med 2009, 206:2897-2906

13. Kreier F, Kalsbeek A, Sauerwein HP, Fliers E, Romijn JA, Buijs RM: "Diabetes of the elderly" and type 2 diabetes in younger patients: possible role of the biological clock. Exp Gerontol 2007, 42:22-27

14. Ando H, Fujimura A: [Circadian clock disruption and diabetes mellitus] Japanese. Nihon Rinsho 2013, 71:2114-2118 
15. Jadhav V, Luo Q, M Dominguez J II, Al-Sabah J, Chaqour B, Grant MB, Bhatwadekar AD: Per2-mediated vascular dysfunction is caused by the upregulation of the connective tissue growth factor (CTGF). PLoS One 2016, 11:e0163367

16. Bhatwadekar AD, Yan Y, Qi X, Thinschmidt JS, Neu MB, Li Calzi S, Shaw LC, Dominiguez JM, Busik JV, Lee C, Boulton ME, Grant MB: Per2 mutation recapitulates the vascular phenotype of diabetes in the retina and bone marrow. Diabetes 2013, 62:273-282

17. Yano M, Iwama A, Nishio H, Suda J, Takada G, Suda T: Expression and function of murine receptor tyrosine kinases, TIE and TEK, in hematopoietic stem cells. Blood 1997, 89:4317-4326

18. Bhatwadekar A, Glenn JV, Figarola JL, Scott S, Gardiner TA, Rahbar S, Stitt AW: A new advanced glycation inhibitor, LR-90, prevents experimental diabetic retinopathy in rats. $\mathrm{Br} \mathrm{J}$ Ophthalmol 2008, 92:545-547

19. Zheng L, Gong B, Hatala DA, Kern TS: Retinal ischemia and reperfusion causes capillary degeneration: similarities to diabetes. Invest Ophthalmol Vis Sci 2007, 48:361-367

20. Anea CB, Zhang M, Stepp DW, Simkins GB, Reed G, Fulton DJ, Rudic RD: Vascular disease in mice with a dysfunctional circadian clock. Circulation 2009, 119:1510-1517

21. Davis S, Aldrich TH, Jones PF, Acheson A, Compton DL, Jain V, Ryan TE, Bruno J, Radziejewski C, Maisonpierre PC, Yancopoulos GD: Isolation of angiopoietin-1, a ligand for the TIE2 receptor, by secretion-trap expression cloning. Cell 1996, 87: $1161-1169$

22. Sato TN, Qin Y, Kozak CA, Audus KL: Tie-1 and tie-2 define another class of putative receptor tyrosine kinase genes expressed in early embryonic vascular system. Proc Natl Acad Sci U S A 1993, 90: 9355-9358

23. Schnurch H, Risau W: Expression of tie-2, a member of a novel family of receptor tyrosine kinases, in the endothelial cell lineage. Development 1993, 119:957-968

24. Kisanuki YY, Hammer RE, Miyazaki J, Williams SC, Richardson JA, Yanagisawa M: Tie2-Cre transgenic mice: a new model for endothelial cell-lineage analysis in vivo. Dev Biol 2001, 230:230-242
25. Arai F, Hirao A, Ohmura M, Sato H, Matsuoka S, Takubo K, Ito K, Koh GY, Suda T: Tie2/angiopoietin-1 signaling regulates hematopoietic stem cell quiescence in the bone marrow niche. Cell 2004, 118 : $149-161$

26. Hangai M, Yoshimura N, Hiroi K, Mandai M, Honda Y: Inducible nitric oxide synthase in retinal ischemia-reperfusion injury. Exp Eye Res 1996, 63:501-509

27. Du Y, Smith MA, Miller CM, Kern TS: Diabetes-induced nitrative stress in the retina, and correction by aminoguanidine. J Neurochem 2002, 80:771-779

28. Anea CB, Cheng B, Sharma S, Kumar S, Caldwell RW, Yao L, Ali MI, Merloiu AM, Stepp DW, Black SM, Fulton DJ, Rudic RD: Increased superoxide and endothelial NO synthase uncoupling in blood vessels of Bmal1-knockout mice. Circ Res 2012, 111:1157-1165

29. Westgate EJ, Cheng Y, Reilly DF, Price TS, Walisser JA, Bradfield CA, FitzGerald GA: Genetic components of the circadian clock regulate thrombogenesis in vivo. Circulation 2008, 117: 2087-2095

30. Rudic RD, McNamara P, Reilly D, Grosser T, Curtis AM, Price TS, Panda S, Hogenesch JB, FitzGerald GA: Bioinformatic analysis of circadian gene oscillation in mouse aorta. Circulation 2005, 112: $2716-2724$

31. Mendez-Ferrer S, Lucas D, Battista M, Frenette PS: Haematopoietic stem cell release is regulated by circadian oscillations. Nature 2008, 452:442-447

32. Tay J, Levesque JP, Winkler IG: Cellular players of hematopoietic stem cell mobilization in the bone marrow niche. Int J Hematol 2017, 105:129-140

33. Prieto P, Fernandez-Velasco M, Fernandez-Santos ME, Sanchez PL, Terron V, Martin-Sanz P, Fernandez-Aviles F, Bosca L: Cell expansion-dependent inflammatory and metabolic profile of human bone marrow mesenchymal stem cells. Front Physiol 2016, 7:548

34. Roson-Burgo B, Sanchez-Guijo F, Del Canizo C, De Las Rivas J: Insights into the human mesenchymal stromal/stem cell identity through integrative transcriptomic profiling. BMC Genomics 2016, 17: 944 